\title{
Signals and noise in tiny axons
}

What factors limit the minimum diameter of an axon? And what is that minimum diameter? Faisal and colleagues used biophysical theory and stochastic simulations to investigate these questions, and concluded that ion-channel noise sets a lower limit of $\sim 0.1 \mu \mathrm{m}$ on the diameter of axons that carry action potentials.

Action potentials are generated and propagated by ion channels, which open and close in response to changes in membrane voltage. However, the opening and closing of these channels is probabilistic, which means that random channel events introduce channel noise into the system. Because the effect of a single $\mathrm{Na}^{+}$ channel on the membrane potential of an axon increases with decreasing axon diameter, in very thin axons the random opening of a single channel could, in theory, generate a spontaneous action potential. However, it

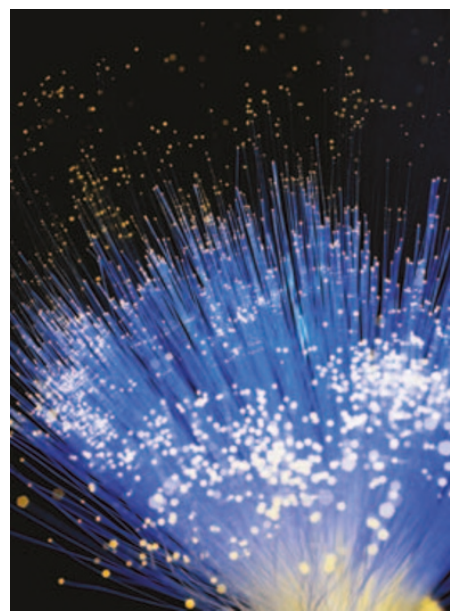

is hard to record from the thinnest axons in the nervous system, so it has been difficult to determine the importance of this effect.

The authors investigated this issue by using stochastic simulations of axons. These simulations draw on empirical data on the responses of single channels to generate models of how a system containing these channels will behave. Simulating action potentials in axons is very demanding, but the authors developed a stochastic simulator, called Modigliani, that uses special routines to tackle this challenge.

Initially, the authors simulated the behaviour of two types of axon - pyramidal cell axons, which form most connections in cortical networks, and the squid axon, which has been extensively studied. They investigated how the generation of spontaneous action potentials by channel noise varied with axon diameter. Despite the differences between the two types of axon, both began to produce significant numbers of spontaneous action potentials at a critical diameter of about 0.15$0.30 \mu \mathrm{m}$ - considerably lower than previous estimates. Below this point, the number of spontaneous action potentials increased exponentially as axon diameter decreased further.

Additional analysis and theoretical work led the authors to conclude that the critical diameter of an axon is relatively insensitive to its biophysical properties, and that most axons will have similar critical diameters - of the order of about 0.1 $\mu \mathrm{m}$. When they studied published electron micrographs of axons from various species, Faisal and colleagues found that the minimum diameter of most axons was about $0.1 \mu \mathrm{m}$, with a few exceptions that might represent developing axons. However, on the basis of structural considerations alone, it should be possible to construct an axon with a diameter of as little as $0.06 \mu \mathrm{m}$, which indicates that channel noise is the limiting factor when it comes to axon miniaturization. Interestingly, neurites that do not conduct action potentials, such as those of Drosophila amacrine cells, can be as small as $0.05 \mu \mathrm{m}$ in diameter, which indicates that the 0.1 $\mu \mathrm{m}$ limit applies only to axons that conduct action potentials.

Further simulations using this or similarly powerful systems should shed more light on the importance of channel noise in neuronal function, and on how the properties of axons might be fine-tuned to optimize signalling reliability.

Rachel Jones

References and links

ORIGINAL RESEARCH PAPER Faisal, A. A. et al Ion-channel noise places limits on the

miniaturization of the brain's wiring. Curr. Biol. 15, 1143-1149 (2005)

FURTHER READING White, J. A. et al. Channel noise in neurons. Trends Neurosci. 23, 131-137 (2000)

WEB SITES

Laughlin's laboratory: http://www.zoo.cam. ac.uk/zoostaff/laughlin/index.html White's laboratory: http://www.bu.edu/ndl/ The Modigliani simulation framework: http:// www.modigliani.co.uk/ 